\title{
Active Stabilization of a Chaotic Urban System
}

\author{
GÜNTER HAAG *, TILO HAGEL and TIMM SIGG \\ Institute of Theoretical Physics, University of Stuttgart, Pfaffenwaldring 57/III, D-70550 Stuttgart, Germany
}

(Received 9 October 1996)

\begin{abstract}
A new method to stabilize dynamical systems by forcing the system variables into the desired unstable stationary point is proposed. The key conception of the method is based on parametric perturbation. This means that the equations of motion are influenced by continuous variation of some selected parameters. Thus - without using any external forces - the motion of the system approaches the chosen unstable stationary point. The variation of the parameters will vanish after the successful stabilization. Therefore, the system and its parameters are changed during the control process only. The algorithm is applied to an urban system within a metropolitan area obeying a Lorenz-type dynamics as well as to the Hénon attractor as an example for a discrete scenario.
\end{abstract}

Keywords: Chaos, Stabilization, Control, Urban system, Town

\section{INTRODUCTION}

The stabilization of dynamic systems behaving chaotically in the absence of an active control provides a challenge for theoretical and experimental research (Schuster, 1984; Weidlich and Haag, 1983).

In general, for a wide class of dynamic systems, chaotic behaviour is undesired because of its lack of predictability concerning the future evolution of the system's trajectory.

Especially in the field of economics and sociology, the "costs" related to chaos have been frequently discussed (Lorenz, 1989; Gabisch and
Lorenz, 1989). Those costs must be considered by the economic agents. Hence, methods or algorithms to avoid or control chaotic behaviour are highly welcome (Shinbrot et al., 1990; Pyragas, 1992).

A few years ago, in 1990, Ott, Grebogi and Yorke. (OGY) proposed a method (Ott et al., 1990) to control chaotic systems. However, this method allows to control a chaotic system by varying at least one accessible parameter. Concerning e.g. a chaotically behaving commodity market the advertising budget of a commodity or the price of the considered commodity are possible influence parameters. Applying the OGY-method,

* Corresponding author. 
the system can be stabilized on an unstable periodic orbit embedded in the chaotic attractor.

The control is carried out by using a small time-dependent change of the current parameter value. A variation of only a few percent of the corresponding parameter is sufficient in order to prevent chaos and to lock in the system onto a predictable dynamical mode. As there are always several periodic orbits available, in the OGYmethod the system can be switched by appropriate market strategies between different types of periodic motion (period one, period two, etc.), and the optimal solution can be chosen.

Although the OGY-method proved its applicability and reliability in several physical, biological and chemistrial applications (Hunt, 1991; Ditto et al., 1990; Garfinkel et al., 1992; Parmanada et al., 1993) the method is still not efficient for socio-economical applications since one has to wait until the trajectory reaches the vicinity of the unstable periodic orbit. The "waitingtime" could be inadequately long. Of course this reduces the field of application to fast evolving systems, which approach the unstable periodic orbit frequently. For slowly evolving systems the OGY-method cannot be recommended.

The aim of this paper is to present a new control mechanism to achieve efficient control even for slowly evolving systems. These systems will be stabilized on an unstable fixed point by an active adjustment of system parameters. As a consequence the system's trajectory is driven from a chaotic to a stationary (time-independent) state.

The theoretical treatment of the method is described in Section 2. In Section 3 the method is applied to an urban system within a metropolitan area. The system dynamics is described by the mean output of the urban system, the number of residents, the land rent, and it obeys a Lorenztype dynamics. Section 4 treats the application to the discrete Hénon attractor in an equivalent manner.

Section 5 gives a brief summary of the results as well as an outlook to further research.

\section{THE METHOD}

We consider a continuous-time chaotic system:

$$
\frac{\mathrm{d} \mathbf{x}(t)}{\mathrm{d} t}=\mathbf{R}(\mathbf{x}, \mathbf{p}) .
$$

$\mathbf{x}$ is the state vector of the model now describing our system. $\mathbf{p}$ is a set of model parameters. Model (1) is assumed to possess at least one unstable stationary point. If the system exhibits several unstable stationary points, one can select the point with the highest accordance to the desired system properties. The chosen unstable stationary point is denoted by $\mathbf{x}_{\mathrm{u}}$ :

$$
\frac{\mathrm{d} \mathbf{x}(t)}{\mathrm{d} t}=0=\mathbf{R}\left(\mathbf{x}_{\mathrm{u}}, \mathbf{p}_{0}\right) .
$$

In order to get a relationship between the control parameters $\mathbf{p}$ and the system's dynamics $\mathbf{x}$ we expand the model in a Taylor series with respect to the parameters $\mathbf{p}$ around the initial parameter values $\mathbf{p}_{0}$

$$
\begin{gathered}
\dot{\mathbf{x}}=\mathbf{R}\left(\mathbf{x}, \mathbf{p}_{0}\right)+ \\
+\left.\frac{\partial \mathbf{R}}{\partial p_{k}}\right|_{\mathbf{x}, \mathbf{p}_{0}} \delta p_{k}+\left.\frac{1}{2} \frac{\partial^{2} \mathbf{R}}{\partial p_{k} \partial p_{l}}\right|_{\mathbf{x}, \mathbf{p}_{0}} \delta p_{k} \delta p_{l} \\
+\cdots ; \quad k, l=1,2, \ldots, M,
\end{gathered}
$$

where $\delta \mathbf{p}=\mathbf{p}-\mathbf{p}_{0} . \mathbf{p}=\left(p_{1}, \ldots, p_{M}\right)^{\mathrm{T}}$ is an $M$ dimensional set of adjustable parameters with $M \leq N$. The active control of the chaotic evolution is performed by appropriately changing one or several control parameters $p_{i}$ of the system in time.

In order to force the trajectory towards the chosen stationary point we require

$$
\dot{\mathbf{x}} \|-\delta \mathbf{x}
$$

where $\delta \mathbf{x}=\mathbf{x}-\mathbf{x}_{\mathrm{u}}$ is the distance vector between the system's current state $\mathbf{x}$ and the unstable stationary point $\mathbf{x}_{\mathrm{u}}$. In other words $\dot{\mathbf{x}}$ is required being parallel to $-\delta \mathbf{x}$. Condition (4) guarantees 
that the system will approach the selected stationary point upon the shortest path. Condition (4) can be rewritten in the form

$$
\dot{\mathbf{x}}(\mathbf{p}) \stackrel{!}{=}-r \cdot \delta \mathbf{x}, \quad r>0,
$$

where the scale factor $r$ can be interpreted as a measure of control velocity. The higher $r$ the faster the stationary point will be reached. Considering only the linear terms of (3) together with (5) we obtain

$$
-r \cdot \delta \mathbf{x}=\mathbf{R}\left(\mathbf{x}, \mathbf{p}_{0}\right)+\left.\frac{\partial \mathbf{R}}{\partial p_{k}}\right|_{\mathbf{x}, \mathbf{p}_{0}} \delta p_{k} .
$$

It is now convenient to introduce the matrix $W_{i k}=\partial R_{i} /\left.\partial p_{k}\right|_{\mathbf{x}, \mathbf{p}_{0}}$ in (6) yielding

$$
W_{i k} \cdot \delta p_{k}=-r \delta x_{i}-R_{i}\left(\mathbf{x}, \mathbf{p}_{0}\right) .
$$

In order to solve (7), one has to find a $(M \times N)$ matrix with the requirement

$$
\sum_{i=1}^{N} S_{l i} W_{i k}=\delta_{l k}
$$

$\delta_{l k}$ is the Kronecker symbol:

$$
\delta_{l k}= \begin{cases}1 ; & l=k \\ 0 ; & l \neq k\end{cases}
$$

Having $\quad M=N$ one finds $S_{l i}=W_{l i}^{-1}, \operatorname{det}\left(W_{l i}\right)$ $\neq 0$. One finally obtains an equation for the determination of the required change of the control parameters

$$
\delta p_{k}=W_{i k}^{-1}\left\{-r \delta x_{i}-R_{i}\left(\mathbf{x}, \mathbf{p}_{0}\right)\right\},
$$

where $r$ is still of free choice. We want to stress that $\delta p_{k}$ vanishes for $\mathbf{x}$ approaching $\mathbf{x}_{\mathrm{u}}$.

Eq. (10) fully determines how one has to change the parameters $p_{0}$ in order to fulfill condition (5). Adjusting the parameter $\mathbf{p}=\mathbf{p}_{0}+\delta \mathbf{p}$ according to (10) the trajectory sets course for $\mathbf{x}_{\mathrm{u}}$. One can use $r$ to minimize the relative parametrical perturbations:

$$
\begin{aligned}
& H(r):=\sum_{k=1}^{M}\left(\frac{\delta p_{k}}{p_{k}}\right)^{2} \stackrel{!}{=} \min , \\
& H(r) \sim\left(r-r_{\min }\right)^{2} .
\end{aligned}
$$

Of course there can occur that $r_{\min }$ is nonpositive, but this is in contradiction to (5) where we presumed $r$ being positive, which is the reason for choosing $r>0$, e.g. $r \in(0,1]$. This is done to again guarantee small perturbations and a nonvanishing control velocity $r$.

Adjusting the parameters in the above described manner we come to the final equations of motion

$$
\dot{\mathbf{x}}=\mathbf{R}\left(\mathbf{x}, \mathbf{p}_{0}+\delta \mathbf{p}\right)
$$

for the system's state vector.

\section{APPLICATION TO AN URBAN SYSTEM}

The method will be illustrated using an example from the field of urban dynamics (Zhang, 1991). An urban system within a metropolitan area is considered. In comparison to the metropolitan area the urban system is assumed to be very small with respect to the economic variables. In other words any change in economic conditions in the urban system will not affect the metropolitan area. Since the short-term dynamics of the urban system is of interest, the metropolitan area can be treated as a stationary environment. It is assumed that locational characteristics of the urban area can be described by the following variables:

$X$, deviation from the mean output of the urban system,

$Y$, deviation from the mean number of residents,

$Z$, land rent. 
The dynamics of the urban system is based on the following reasonable assumptions:

- The rate of change of the deviation from the mean urban output $\mathrm{d} X / \mathrm{d} t$ is proportional to the deviation in excess demand $\left(a_{2} Y-a_{3} X\right)$, where $a_{2}$ is the per capita demand of the urban output due to residents and $a_{3}$ is the rate at which the urban output is supplied to the urban area.

- The rate of change of the deviation from the mean number of urban residents $\mathrm{d} Y / \mathrm{d} t$ can be separated into two parts. The first part represents the firm's additional demand for labour to produce $c_{2} X$ which is decreased by the additional total supply of labour to the urban labour market $c_{3} Y$. The second part $-c_{4} X Z$ stands for the effect of emigration due to changes in the land rent $Z$. It also takes into account that people prefer to live in places where land rent is low.

- The rate of change in the land rent $\mathrm{d} Z / \mathrm{d} t$ is related to the current rent level $-d_{2} Z$ as well as to the output and the number of residents $d_{1} X Y$.

These assumptions finally yield the following set of non-linear differential equations:

$$
\begin{aligned}
& \frac{\mathrm{d} X}{\mathrm{~d} t^{*}}=a_{1}\left(a_{2} Y-a_{3} X\right), \\
& \frac{\mathrm{d} Y}{\mathrm{~d} t^{*}}=c_{1}\left(c_{2} X-c_{3} Y\right)-c_{4} X Z, \\
& \frac{\mathrm{d} Z}{\mathrm{~d} t^{*}}=d_{1} X Y-d_{2} Z .
\end{aligned}
$$

Appropriate scaling of the parameters

$$
S_{0}=\frac{a_{1} a_{3}}{c_{1} c_{3}}, \quad R_{0}=\frac{a_{2} c_{2}}{a_{3} c_{3}}, \quad B_{0}=\frac{d_{2}}{c_{1} c_{3}},
$$

the time

$$
t=\frac{t^{*}}{c_{1} c_{3}}
$$

and the variables

$$
\begin{aligned}
& x=\sqrt{\frac{c_{4}}{d_{1}}} \frac{d_{1} X}{c_{1} c_{3}}, \\
& y=\sqrt{\frac{c_{4}}{d_{1}}} \frac{d_{1} a_{2} Y}{a_{3} c_{1} c_{3}}, \\
& z=\frac{a_{2} c_{4} Z}{a_{3} c_{1} c_{3}}
\end{aligned}
$$

leads to the Lorenz system (Lorenz, 1963)

$$
\dot{\mathbf{x}}=\mathbf{L}\left(\mathbf{x} ; \mathbf{p}_{0}\right),
$$

or

$$
\begin{aligned}
\dot{x}(t) & =L_{x}(\mathbf{x}, \mathbf{p})=S_{0} y-S_{0} x, \\
\dot{y}(t) & =L_{y}(\mathbf{x}, \mathbf{p})=R_{0} x-y-x z, \\
\dot{z}(t) & =L_{z}(\mathbf{x}, \mathbf{p})=-B_{0} z+x y .
\end{aligned}
$$

Therefore the urban dynamics is described by the trajectory $\mathbf{x}(t)=(x(t), y(t), z(t))$ for given initial conditions $\mathbf{x}\left(t_{0}\right)=\left(x\left(t_{0}\right), y\left(t_{0}\right), z\left(t_{0}\right)\right) . \mathbf{p}_{0}=\left(S_{0}\right.$, $\left.R_{0}, B_{0}\right)$ in (17) represents three real positive parameters introduced in (15). It can be shown that for some parameter values the solution of the Lorenz equations oscillates in a chaotic way, apparently forever. In addition, for certain parameter values "perturbulence" occurs, a phenomenon characterized by chaotically oscillating trajectories for long periods before finally settling down in a stable stationary or stable periodic orbit. Intermittent behaviour can also be identified where trajectories alternate between chaotic and apparently stable behaviour. In Fig. 1 a plot of the Lorenz attractor is provided where $S_{0}=10, R_{0}=28, B_{0}=\frac{8}{3}$.

Except the trivial solution $\left(x_{1}=0, y_{1}=0\right.$, $\left.z_{1}=0\right)$ there exist two more stationary points in the Lorenz system:

$$
\begin{aligned}
& \left(x_{2,3}, y_{2,3}, z_{2,3}\right) \\
& =\left( \pm \sqrt{B_{0}\left(R_{0}-1\right)}, \pm \sqrt{B_{0}\left(R_{0}-1\right)}, R_{0}-1\right) \\
& =( \pm 8.4853, \pm 8.4853,27.0000) .
\end{aligned}
$$




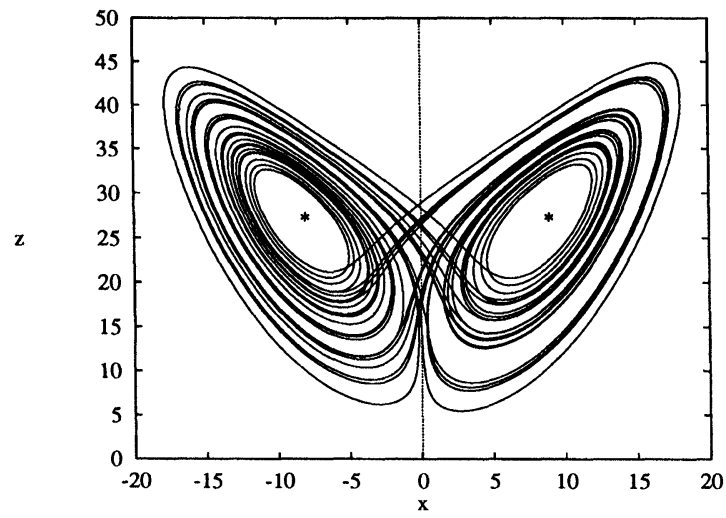

FIGURE 1 Projection of the chaotic attractor into the $(x, \mathrm{z})$-plane. The asterisks mark the stationary points $\mathbf{x}_{2}, \mathbf{x}_{3}$.

Those two symmetric unstable foci are placed in the centres of the two 'Lorenz lobes' (see Fig. 1).

Suppose the maximization of the urban output is used as a selection criterion, the stationary point $\mathbf{x}_{\mathrm{u}}=\left(x_{2}, y_{2}, z_{2}\right)$ will be selected. The stabilization of the system's trajectory on $\mathbf{x}_{\mathbf{u}}$ provides the considerable advantage of stable land rents together with an increased urban output. Additional costs due to uncertain population numbers of residents and related variations in land rent can therefore be prevented.

Corresponding to (10), the necessary adaptation of the parameters $S, R$ and $B$ can be calculated:

$$
\begin{aligned}
& \delta S=\frac{1}{x}\left\{r \delta x+L_{x}\left(\mathbf{x}, \mathbf{p}_{0}\right)\right\} \\
& \delta R=\frac{-1}{x}\left\{r \delta y+L_{y}\left(\mathbf{x}, \mathbf{p}_{0}\right)\right\} \\
& \delta B=\frac{1}{z}\left\{r \delta z+L_{z}\left(\mathbf{x}, \mathbf{p}_{0}\right)\right\}
\end{aligned}
$$

The stabilizing procedure can now be numerically performed by applying the parameter perturbations (20). In Fig. 2 the stabilization procedure is illustrated. At time $t_{0}$ the chaotic dynamics of the Lorenz system starts on the attractor. Although the parameters can be adjusted at any time we decide to do this only within a sphere of radius $\rho$ centered at $\mathbf{x}_{\mathrm{u}}$. Apart from rendering a better representation this is done in order to

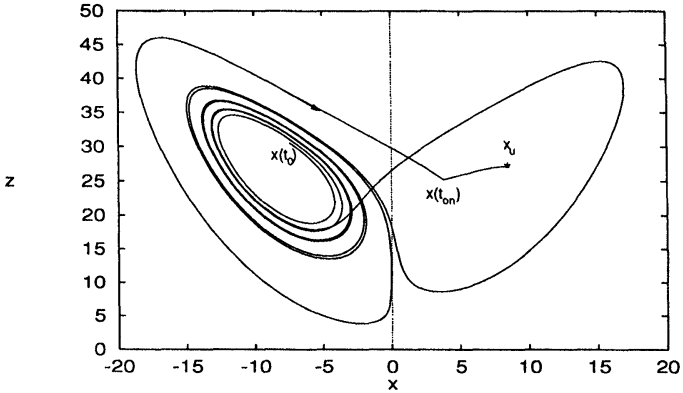

FIGURE 2 Projection of a controlled trajectory into the $(x, z)$-plane. The asterisk marks the stationary point $\mathbf{x}_{\mathrm{u}}$. The control radii $\rho$ around $\mathbf{x}_{\mathrm{u}}$ is 5.5.

avoid too large parameter perturbations. After several chaotic cycles the trajectory enters the sphere and the control mechanism is switched on (time $t_{\text {on }}$ ). The computed time-dependent variation of the control parameters $S, B, R$ force the system's trajectory towards the selected stationary point $\mathbf{x}_{\mathrm{u}}$.

Obviously the stabilizing procedure provides a very efficient method to control deterministic chaos in the field of non-linear dynamics.

In Fig. 3 the necessary relative parameter variations in dependence of the radius $\rho$ of the sphere in space-state is depicted.

Therefore various control radius $\rho$ are selected. For each control radius $\rho$ a set of (100-) random initial conditions was chosen.

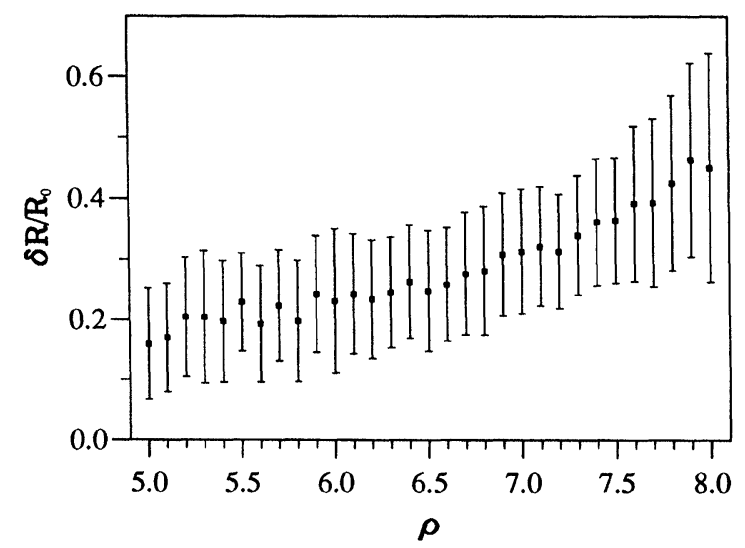

FIGURE 3 Mean values of relative maximum parametrical perturbations $\delta R$. 


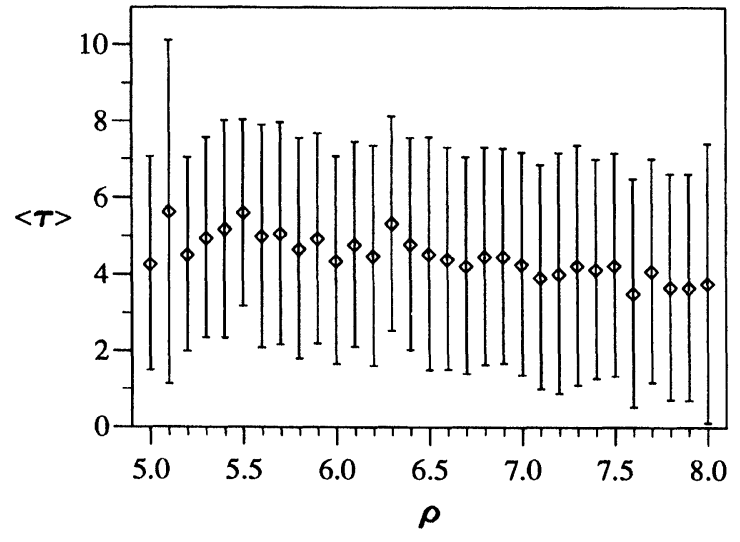

FIGURE 4 Mean values of control time $\tau$.

The maximum parameter perturbation of each run is ascertained and the mean values and standard deviations are plotted (see Fig. 3).

As one can easily see the mean maximum parametrical perturbation decreases with a decreasing control radius $\rho$. This implies that it is less expensive concerning the maximum parametrical perturbation to control the system being in the proximity of $\mathbf{x}_{\mathrm{u}}$. However, one has to take into account an increasing total control time $\left(t_{\text {on }}-t_{0}\right)$.

In the same way the mean control time $\tau$ in dependence of the control radius $\rho$ is shown in Fig. 4.

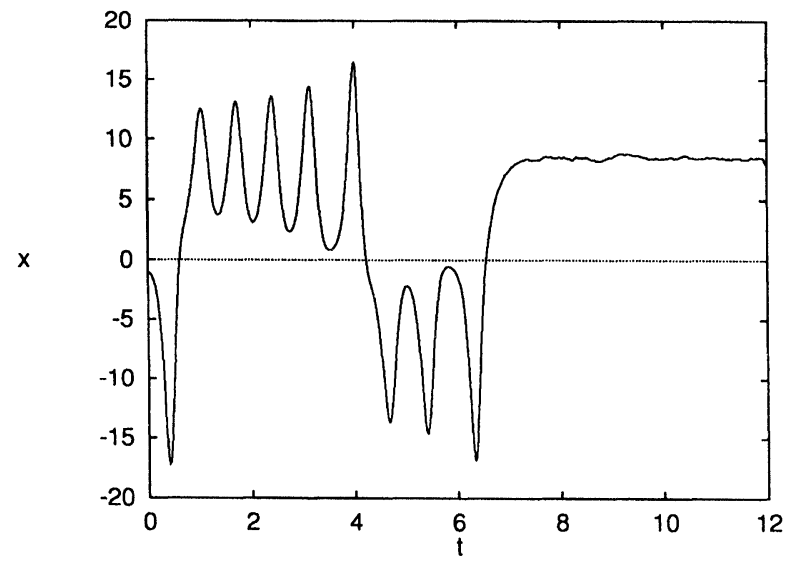

FIGURE 5 Time dependence of the $z$-component with noise $(\alpha=0.1)$. The control was first activated at $t=6.56$ and $\mathbf{x}_{\mathrm{u}}$ was finally reached at $t \approx 7.6$.
The slightly with $\rho$ decreasing mean control time has to be seen in relation to the increase of the relative parameter variation necessary to bring the trajectory to $x_{\mathrm{u}}$.

It is a fundamental question whether or not the stabilizing procedure also works in the presence of noise related, e.g. to uncertain environmental conditions.

In Fig. 5 the performance of the method in the presence of delta-correlated gaussian noise $\mathbf{K}$

$$
\begin{aligned}
\left\langle K_{i}\left(t_{m}\right) K_{j}\left(t_{n}\right)\right\rangle & =\alpha \delta_{i j} \delta\left(t_{n}-t_{m}\right) \\
\left\langle K_{i}\right\rangle & =0
\end{aligned}
$$

is investigated, where $\alpha$ is the strength of the noise. For this aim the fluctuation force $\mathbf{K}$ is added in (17).

Figure 5 shows that the method still works very well for $\alpha=0.1$. The system can be stabilized even against this noise level. Of course the deviation of the state vector $\left(\mathbf{x}_{\text {noise }}\right)$ caused by the strength of the noise $\alpha$ must be smaller than the control radius $\rho$ (see Fig. 6)

$$
\frac{\left|\mathbf{x}_{\mathrm{u}}-\mathbf{x}_{\text {noise }}\right|}{\rho}<C,
$$

where $C$ depends on the noise spectrum and on the required efficiency of the control mechanism.

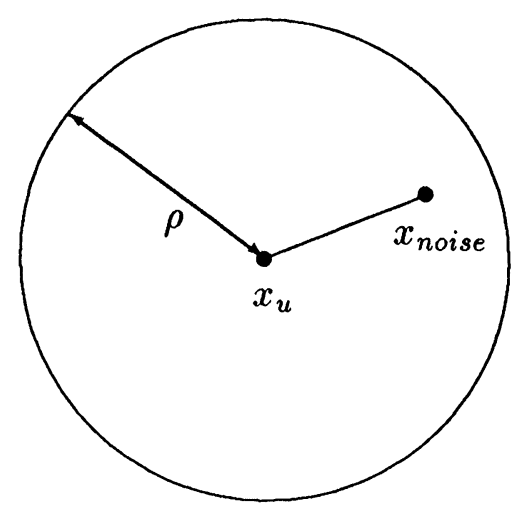

FIGURE 6 The effect of noise on the stabilization procedure. 


\section{APPLICATION TO THE HÉNON ATTRACTOR}

The one-dimensional iterative equation (Hénon, 1976)

$$
x_{n+1}=a-\left(x_{n}\right)^{2}+b x_{n-1}
$$

with positive $a, b$ is well known. It is the Hénon map. Figure 7 shows the corresponding strange attractor with the numerical values $a=1.29$ and $b=0.3$.

The proposed method is extended to the case of discrete dynamics and is applied to the Hénon map. The Hénon map possesses two fixed points $x_{1 / 2}^{*}$ which satisfy the relation

$$
x_{1 / 2}^{*}=x_{n+1}=x_{n}=x_{n-1} .
$$

They read

$$
x_{1 / 2}^{*}=\frac{1}{2}\left(b-1 \pm \sqrt{(1-b)^{2}+4 a} .\right.
$$

For stabilization we choose without loss of generality $x_{1}^{*}$ and call it $x_{\mathrm{F}}$. It is convenient to extend the system on two dimensions and to rewrite (23):

$$
\begin{aligned}
& x_{n+1}=a-\left(x_{n}\right)^{2}+b y_{n}, \\
& y_{n+1}=x_{n} .
\end{aligned}
$$

The discrete equations (26) correspond to the continuous-time system (1). But in the discrete case the demand

$$
x_{n+1}-x_{n} \stackrel{!}{=}-\delta x
$$

replaces (5). Eq. (27) inserted into (26) leads to

$$
-\delta x=a-\left(x_{n}\right)^{2}-x_{n}+b y_{n} .
$$

By choosing $b$ as parameter of control one obtains in correspondence to (10) the determination of the required change of the control parameter

$$
\delta b=\frac{1}{y_{n}}\left(\left(x_{n}\right)^{2}-x_{n}-a-b_{0} y_{n}-\delta x\right) .
$$

With this adaption of parameter $b$ the stabilizing procedure can now be performed.

In Fig. 8 a trajectory of the Hénon system is shown. The method starts working at $n=1200$. The numerical value of the control radius was chosen as $\rho=0.001$.

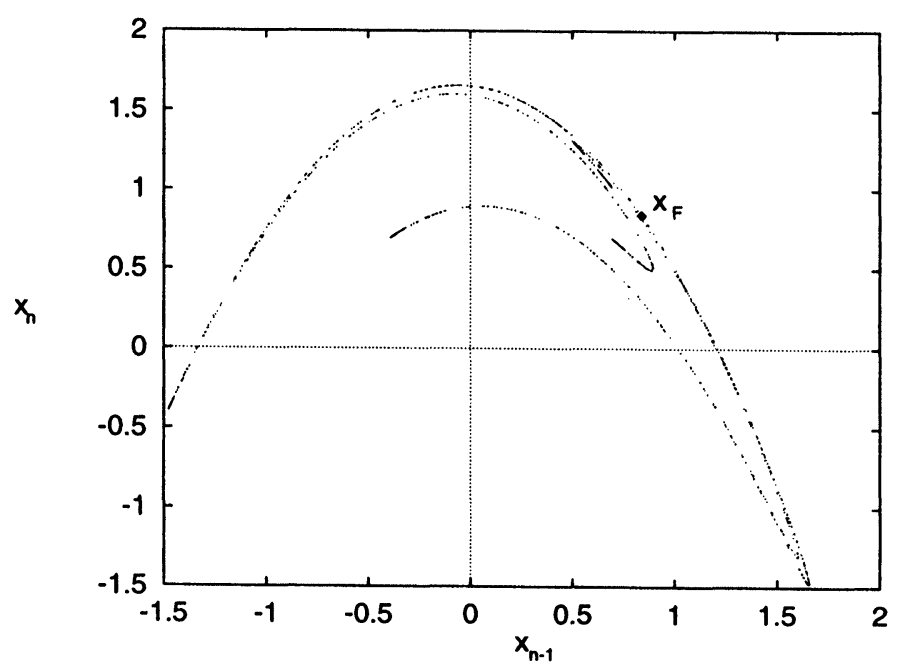

FIGURE 7 The Hénon attractor in two-dimensional mapping. One of the fixed points $\left(x_{\mathrm{F}}\right)$ is marked. 


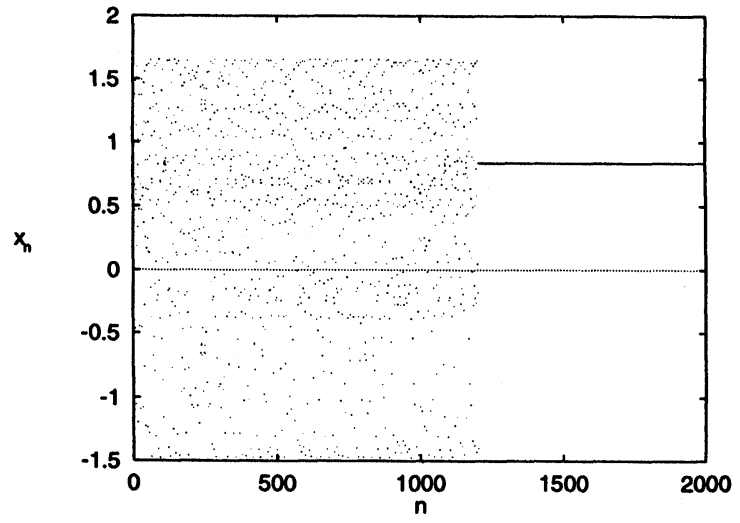

FIGURE 8 The projection of Hénon attractor into one dimension. The mechanism of stabilization starts at $n=1200$.

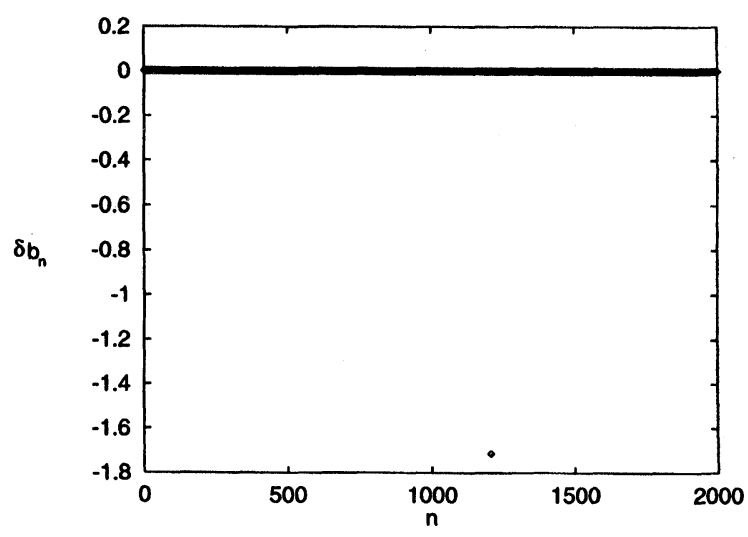

FIGURE 9 Parametrical perturbation $\delta b$ in dependence on $n$.

As it can be seen in Fig. 9 the total control time has only the length of a single iteration step.

\section{CONCLUSION}

The proposed method is rather general. It is applicable to time-continuous systems as well as to time-discrete systems. It can be seen that the method is very efficient for controlling systems either based on sets of differential equations or on iterative maps. It has been shown that even in the presence of noise a stabilization of chaotic trajectories can be performed. The reliability of the control mechanism depends on the noise spectrum and the chosen control radius. In case of an application to the Hénon system the control parameter adaption had only once a nonnegligible value.

With increasing control radius the required parameter variation also increases whereas the control time decreases only slightly.

The method is exemplified on the one hand on an urban system obeying a Lorenz dynamics and on the other hand to the Hénon system. The urban output and the urban population could be stabilized on the desired higher values.

\section{References}

W.L. Ditto, S.N. Rauseo and M.L. Spano, Phys. Rev. Lett. 65, 3211 (1990).

G. Gabisch and H.-W. Lorenz, Business Cycle Theory (Springer-Verlag, Berlin, Heidelberg, New York, 1989).

A. Garfinkel, M.L. Spano, W.L. Ditto and J.N. Weiss, Science 257, 1230 (1992).

M. Hénon, A two-dimensional mapping with a strange attractor, Commun. Math. Phys. 50, 69 (1976).

E.R. Hunt, Phys. Rev. Lett. 67, 1953 (1991)

E.N. Lorenz, J. Atmos. Sci. 20, 130 (1963).

H.-W. Lorenz, Nonlinear Dynamical Economics and Chaotic Motion (Springer-Verlag, Berlin, Heidelberg, 1989).

E. Ott, C. Grebogi and J.A. Yorke, Phys. Rev. Lett. 64, 1196 (1990).

P. Parmanada, P. Shepard, R.W. Rollins and H.D. Dewald, Phys. Rev. A 47, 3003 (1993).

K. Pyragas, Phys. Lett. A 170, 421 (1992).

H.G. Schuster, Deterministic Chaos (Physik-Verlag, Weinheim, 1984).

T. Shinbrot, E. Ott, C. Grebogi and J.A. Yorke, Using Chaos to Direct Trajectories to Targets, Phys. Rev. Lett. 65, 3215 (1990).

W. Weidlich and G. Haag, Concepts and Models of a Quantitative Sociology (Springer-Verlag, Berlin, 1983).

W. Zhang, Synergetic Economics: Time and Change in Nonlinear Economics (Springer-Verlag, Berlin, Heidelberg, New York, 1991). 


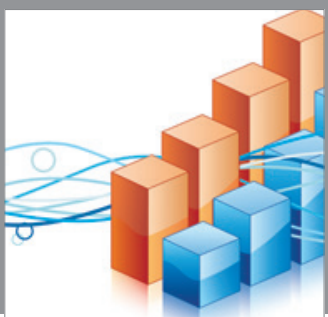

Advances in

Operations Research

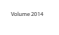

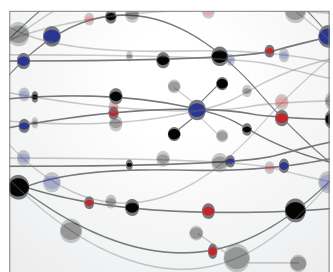

\section{The Scientific} World Journal
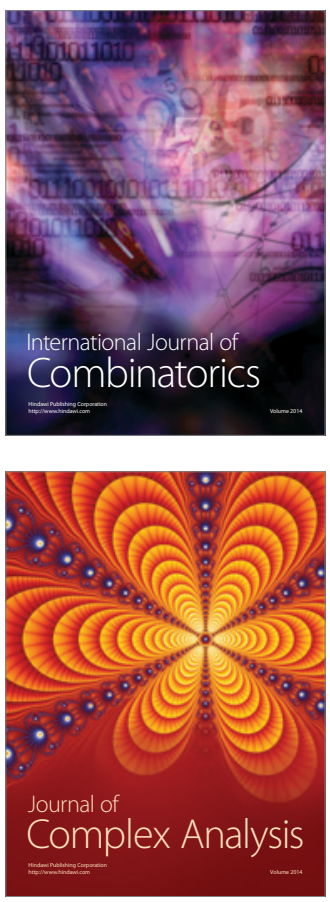

International Journal of

Mathematics and

Mathematical

Sciences
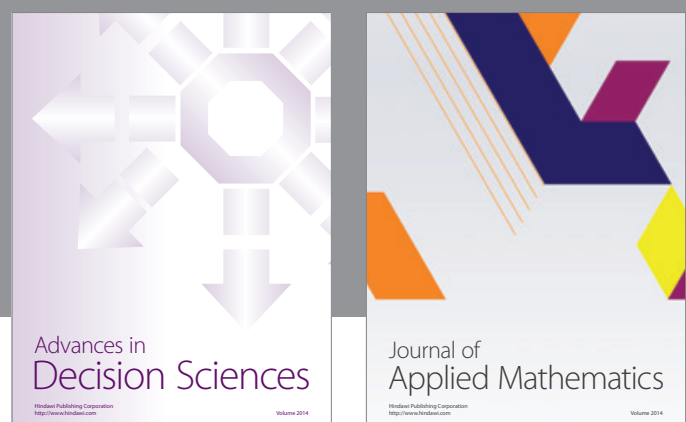

Journal of

Applied Mathematics
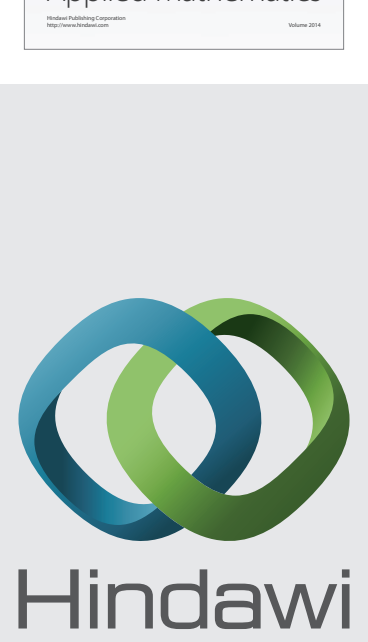

Submit your manuscripts at http://www.hindawi.com
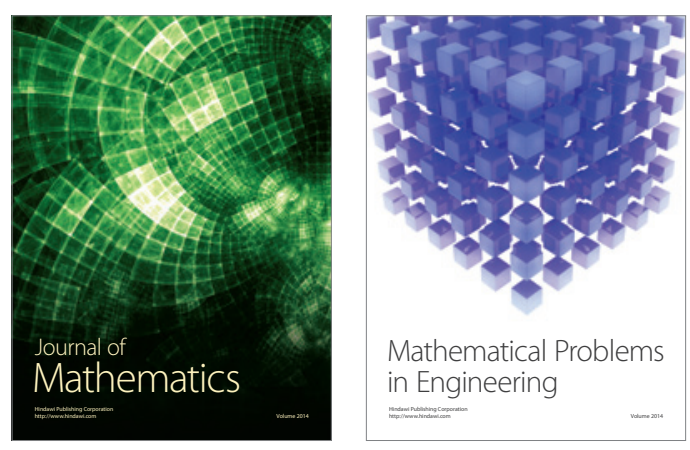

Mathematical Problems in Engineering
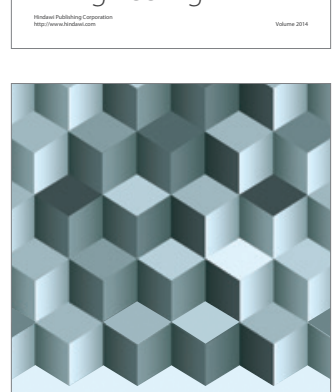

Journal of

Function Spaces
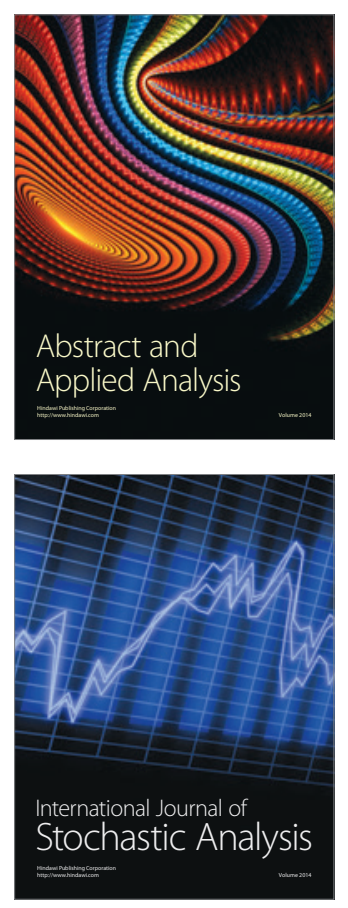

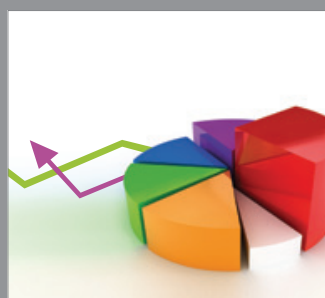

ournal of

Probability and Statistics

Promensencen
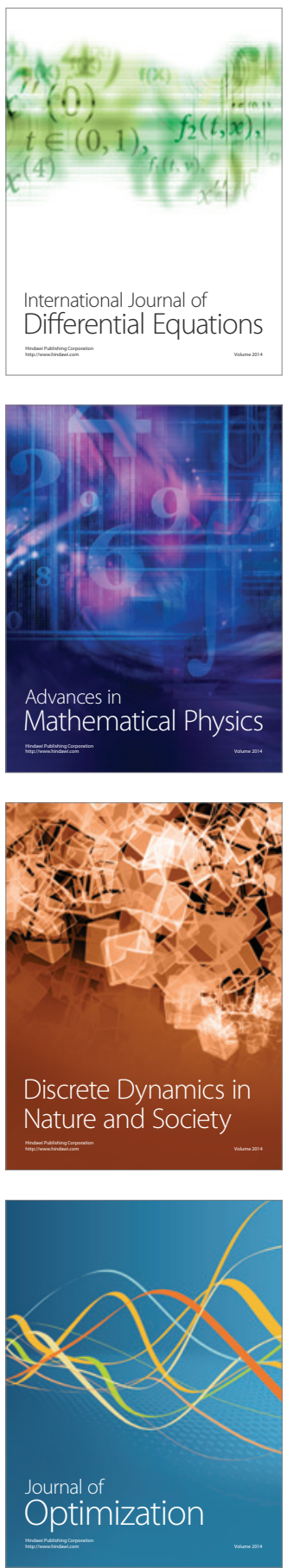